\title{
Tratamiento contable de la provisión por cierre de minas
}

\author{
Pedro P. Franco Concha \\ Magister en Gestión Ambiental para el Desarrollo Sostenible \\ por La Pontificia Universidad Javeriana, Bogotá, Colombia. \\ Master in Business Administration por el Kogod College \\ Business School, The American University. Profesor, Consultor \\ e Investigador. Universidad del Pacífico, Lima Perú. \\ Maria Elena Angulo \\ Rosa Cáceres \\ Isabel Yamashiro
}

\section{Resumen}

La Ley del Impuesto a la Renta vigente y su Reglamento no mencionan ni reconocen a la Provisión por Cierre de Minas ${ }^{1}$ como gasto deducible para efectos del cálculo de la renta neta imponible ${ }^{2}$, lo que genera un vacío legal que no permite que las empresas que se dedican a la actividad minera puedan determinar si dicha provisión se debería o no considerar como gasto deducible del Impuesto a la Renta.

La presente investigación tiene como objetivo sustentar y validar la especificación e inclusión o no de la Provisión por Cierre de Minas como gasto deducible para efectos de la Ley del Impuesto a la Renta a través de la evaluación del tratamiento contable financiero y tributario de dicha provisión.

\section{Palabras clave:}

Impuesto a la Renta, Minería, Cierre de Minas, NIC 16, Perú.

1. Por provisión, por Cierre de Minas se hace alusión al reconocimiento en los Estados Financieros actuales o registro contable de los gastos futuros en que se incurrirá para llevar a cabo el Plan de Cierre de Minas.

2. De acuerdo con el TUO de la Ley del Impuesto a la Renta, Artículos $20^{\circ}, 57^{\circ}$ y $58^{\circ}$; y el Reglamento de la Ley del Impuesto a la Renta, Inciso d) del artículo $11^{\circ}$, Incisos a) y b) del artículo 310: La Renta Neta Imponible se obtiene de la Renta bruta, menos gastos deducibles, más otros ingresos gravables, menos pérdida tributaria compensable de ejercicios anteriores. 


\section{Introducción}

Antes del año $1993^{3}$, la actividad minera, sin un marco legal adecuado, generó muchos problemas de contaminación, fundamentalmente de los cursos de las aguas de los ríos y de los lagos, alterando el paisaje y generando problemas sociales de gran envergadura 4 .

El Plan de Cierre de Minas es considerado importante para el país, porque regula la actividad minera con la finalidad de que se desarrolle de manera sostenible. De esta manera se previene posibles conflictos con el entorno social. Este plan es un instrumento de gestión ambiental integrado por acciones técnicas y legales que va a permitir a los titulares de la actividad minera hacer las provisiones económicas necesarias para invertir y rehabilitar las áreas alteradas ${ }^{5}$.

Siendo el Plan de Cierre de Minas un elemento primordial para la operación de cualquier unidad minera, es necesario cuestionar el tratamiento contable tributario de la Provisión por Cierre de Minas vigente y confrontar el razonamiento que lo sustenta contra el del tratamiento contable financiero, el cual admite dicha provisión como gasto en el Estado de Resultados o Estado de Ganancias y Pérdidas.

Asimismo, cabe mencionar que si bien la Ley del Impuesto a la Renta permite deducir los gastos relacionados al Cierre de Minas, esta lo permite solo en el período en el que dichos gastos son incurridos; sucediendo la mayor cantidad en las etapas de Cierre Final y Post Cierre. En estas etapas finales de las operaciones, las unidades mineras ya no generan el suficiente nivel de renta como para poder beneficiarse de la deducción de la totalidad de los gastos mencionados ${ }^{6}$.

\section{Cierre de Minas}

El presente capítulo expone el tema del Cierre de Minas, tema que es necesario conocer para poder desarrollar el presente trabajo de investigación. Cuenta con un subcapítulo introductorio al tema, en el cual se presenta el ciclo de vida de las minas, la definición de Cierre de Minas y el proceso de cierre, principalmente. Asimismo, el segundo subcapítulo presenta el Plan de Cierre de Minas, el cual constituye el componente más importante de la Ley de Cierre de Minas; en este subcapítulo se contempla la definición, objetivo, y etapas del plan, entre otros.

\section{Introducción al Cierre de Minas}

\section{Ciclo de vida de una mina}

La comprensión del Cierre de Minas involucra entender el ciclo de vida total de la mina ${ }^{7}$. El aprovechamiento de los recursos mineros pasa por las etapas de exploración, construcción, operación y cierre. No se avanza a una siguiente etapa hasta no haber culminado la anterior ${ }^{8}$.

Una mina, además, puede tener varias zonas productivas y diversas actividades dentro de toda su área geográfica. Cuando una de estas deja de operar se procede a su cierre, considerándose dicha acción como un "cierre progresivo»". Así, en paralelo a las etapas del ciclo de vida de la mina se desarrollan programas progresivos de cierre de la misma ${ }^{10}$.

3. Hasta el año 1993 había una ausencia de normatividad en materia ambiental para las actividades mineras. A partir de ese momento, se implantó un proceso de control y prevención de afectación ambiental, que obliga a los responsables de los proyectos mineros a presentar un Estudio de Impacto Ambiental, que debe ser aprobado antes del inicio de actividades. Según: Ministerio de Energía y Minas, Nota de prensa: «Proponen alianzas estratégicas para su remediación. Hay 850 pasivos ambientales en el país, que son herencia de la vieja minería», junio 2006. En: http://www.minem.gob.pe/common/prensa/data/notihoy1800.pdf.

4. Congreso de la República 2003b.

5. Ibídem.

6. De Orbegoso 2004.

7. CIDA - Proyecto de asistencia al sector público minero metalúrgico del Perú, Oficina de Evaluación Ambiental de British Columbia Golder Associates LTD. 2002.

8. Sociedad Nacional de Minería, Petróleo y Energía 2005.

9. Ver 3.1.5 Los diferentes escenarios del Cierre de Minas.

10. Sociedad Nacional de Minería, Petróleo y Energía 2005. 


\section{Consideraciones para el Cierre de Minas}

Es importante entender que la introducción de nuevas actividades mineras en un área ocasionará inevitablemente cambios considerables tanto a la tierra como a la gente relacionada a ella. $Y$, mientras la vida de la mina tiende a ser relativamente corta (operando durante 10 a 50 años), los cambios al medio ambiente y a la sociedad producidos por las minas persistirán por un tiempo largo' ${ }^{11}$.

\section{Definición de Cierre de Minas}

El Cierre de Minas es el conjunto de actividades realizadas por la empresa minera destinadas a la rehabilitación de las áreas utilizadas por esta ${ }^{12}$ a través del tiempo. Por ello, el Cierre de Minas se desarrolla teniendo en cuenta cada una de las etapas de la vida de una unidad operativa, con actividades particulares como la rehabilitación temporal de áreas durante las operaciones, la reconformación del terreno durante el cierre de la unidad o el monitoreo del agua superficial y subterránea luego del cierre de la unidad, entre otros.

\section{Los diferentes escenarios del Cierre de Minas}

El Cierre de Minas puede presentar los siguientes escenarios ${ }^{13}$ :

- Cierre Temporal:

Como resultado de las circunstancias económicas u operacionales, es posible que las actividades mineras y/o de procesamiento sean temporalmente suspendidas. Por ejemplo, estas pueden ser suspendidas esperando la recuperación del precio de los metales. Un Cierre Temporal de esta naturaleza es normalmente planificado y asume que la operación se reiniciará.

- Cierre Progresivo:

El Cierre Progresivo es un escenario que ocurre de manera simultánea a la etapa de operación de una mina, cuando un componente o parte de un componente de la actividad minera deja de ser útil. Debido a ello deberá ser sometido a actividades de cierre tales como desmantelamiento, demolición, restablecimiento de la forma del terreno, y/o revegetación.

- Cierre Final:

El Cierre Final comienza cuando, a consecuencia del agotamiento de los recursos minerales económicos, cesan las operaciones de minado y de procesamiento. El Cierre Final es la ejecución de las actividades contempladas en el Plan de Cierre Final para cumplir con los objetivos ambientales y sociales específicos.

- Actividades Post Cierre:

Entre las actividades que se efectúan en esta etapa se encuentran el tratamiento de afluentes y emisiones y el monitoreo y mantenimiento que deben realizarse luego de concluidas las acciones de rehabilitación hasta que se demuestre la estabilidad física y química del residuo o componente minero susceptible de generar impactos negativos, de acuerdo con el Plan de Cierre de Minas aprobado $^{14}$.

\section{Plan de Cierre de Minas}

El Plan de Cierre de Minas es un instrumento de gestión ambiental en el que se establecen pautas a ser efectuadas por el titular de la actividad minera (empresa), a fin de rehabilitar las áreas utilizadas por este. Se busca que la rehabilitación alcance características compatibles con un ambiente saludable, seguro y adecuado para el desarrollo de la vida ${ }^{15}$. Conforme se ejecuta el Plan de Cierre de Minas se lleva a cabo la rehabilitación antes mencionada. Asimismo, este plan forma parte de los documentos de Certificación Ambiental con

11. CIDA - Proyecto de asistencia al sector público minero metalúrgico del Perú, Oficina de Evaluación Ambiental de British Columbia Golder Associates LTD. 2002.

12. Sociedad Nacional de Minería, Petróleo y Energía 2005.

13. Ministerio de Energía y Minas, Dirección General de Asuntos Ambientales Mineros 2006.

14. Congreso de la República del Perú 2005.

15. Sociedad Nacional de Minería, Petróleo y Energía 2005. 
los que debe contar toda unidad minera, entre los que se encuentran el Estudio de Impacto Ambiental ${ }^{16}$ (EIA) y el Programa de Adecuación y Manejo Ambiental ${ }^{17}$ (PAMA).

Por otro lado, la elaboración y presentación del Plan de Cierre de Minas es obligatoria para todas las empresas que se dediquen a la actividad minera y que se encuentren en operación, recién inicien operaciones o las reinicie. La elaboración y presentación de este documento también es de carácter obligatorio para las unidades mineras que se encuentren en la etapa de exploración. Asimismo, la presentación del mencionado plan por parte de los responsables de la actividad minera se debe efectuar en el plazo máximo de un año desde la presentación del EIA o PAMA, quedando entendido que este plan debe ser más detallado y completo que el presentado en estos instrumentos de gestión ambiental. Además, la revisión del plan debe efectuarse cada cinco años, con la finalidad de actualizar sus valores o adecuarlos a los avances técnicos, económicos, sociales o ambientales.

El desarrollo del Plan de Cierre de una mina debe establecerse de acuerdo con las características particulares de la mina, por ende, es necesario un análisis de impacto ambiental para identificar y caracterizar los recursos que van a ser protegidos. Una vez que los recursos, que incluyen el agua, el aire, la fauna silvestre y los usos futuros de la tierra, han sido determinados, deben desarrollarse niveles de protección para establecer un uso beneficioso de ellos ${ }^{18}$.

El plan incluye una narración, dibujos de diseño, especificaciones sobre construcción, así como certificación de calidad, monitoreo y otros planes de mantenimiento después del cierre ${ }^{19}$. Además, en lo que respecta a los documentos adicionales que el Plan de Cierre de Minas debe incluir, se encuentran el presupuesto del cierre, el cronograma de su ejecución y las garantías respectivas otorgadas al Estado para asegurar su cumplimiento. Estas últimas están constituidas por el costo del cierre y todos los gastos directos e indirectos que se deriven de la ejecución del plan en cuestión. Asimismo, contablemente se debe efectuar una provisión ${ }^{20}$ por el importe que se constituya como la garantía mencionada (que cubre las etapas de Cierre Final y Post Cierre) así como por los gastos a efectuarse durante el Cierre Progresivo, a fin de que se refleje el total de las futuras erogaciones a efectuarse para poder cumplir con las actividades de rehabilitación consignadas en el Plan de Cierre de Minas. Es decir, este importe provisionado hace referencia al gasto en el que se va a incurrir en cada una de las etapas del Cierre de Minas (Cierre Progresivo, Cierre Final y Post Cierre).

Por otro lado, un requisito fundamental con el que debe contar dicho plan es su aprobación por el Ministerio de Energía y Minas (MEM), a través de la Dirección General de Asuntos Ambientales Mineros (DGAAM). Asimismo, el $M M^{21}$ es la institución encargada de fiscalizar el cumplimiento del plan, así como de ejecutar las sanciones correspondientes. Es importante señalar que la exigencia en el detalle de los planes de cierre será mayor a medida que avance el ciclo de vida de la mina y, por ende, se cuente con una mayor cantidad de información.

El Plan de Cierre debe constar de escenarios conforme la mina sea explotada para que la realización de las actividades que lo detallan sea efectuada de una manera más ordenada y para que se cumpla cabalmente el propósito de dicho plan: ser una herramienta de gestión responsable, oportuna y segura con el medio ambiente para evitar el problema de pasivos ambientales ${ }^{22}$

16. Ley 27446 (20/04/2001). Ley del Sistema Nacional de Evaluación del Impacto Ambiental.

17. DS 016-93-EM (28/04/1993). Reglamento sobre la Protección del Medio Ambiente.

18. Biblioteca de la UNMSM 2008.

19. Ibídem.

20. Esta provisión se reconoce en el momento en el que se efectúan las garantías, es decir, una vez aprobado el Plan de Cierre de Minas. Este hecho puede ocurrir cuando la mina esté en operación, cuando la inicie o la reinicie.

21. Específicamente la Dirección General de Minería de dicho ministerio.

22. Un pasivo ambiental es una situación ambiental que, generada por el hombre en el pasado y con deterioro progresivo en el tiempo, representa actualmente un riesgo al ambiente y la calidad de vida de las personas. 
mineros $^{23}$. De esta manera, las etapas que deben estar presentes en el plan son las siguientes ${ }^{24}$ :

- Plan de Cierre Inicial: Identifica los objetivos principales para el Cierre de Minas con el fin de orientar el desarrollo y diseño del proyecto.

- Plan de Cierre Final: Es la actualización completa de los Planes de Cierre precedentes.

- Evaluación en la etapa Post Cierre: Los informes de la etapa Post Cierre tienen la finalidad de proporcionar una evaluación de la efectividad de las actividades de cierre.

De los temas tratados en el presente capítulo se puede concluir que una de las consideraciones fundamentales para el Cierre de Minas es que la actividad minera genera impactos permanentes y negativos en la zona en la que se desarrolla, por lo que resulta importante prestarle mucha atención al oportuno y correcto emprendimiento del conjunto de actividades que involucran el Cierre de Minas con el fin de evitar la existencia de una mayor cantidad de pasivos ambientales sin resolver. Asimismo, el Plan de Cierre, herramienta en la que se detallan las actividades antes mencionadas, permite conocer anticipadamente los problemas técnicos y sociales, y el costo asociado a la rehabilitación del medio ambiente en un proyecto minero, de modo tal que se pueda proveer oportunamente los recursos para ejecutar el cierre, o en caso contrario no emprender el proyecto ${ }^{25}$.

\section{Ley 28090 - Ley que regula el cierre de minas}

\section{Artículos observados por el Poder Ejecutivo}

El Proyecto de Ley de Cierre de Minas, antes de aprobarse como la Ley 28090 - Ley que regula el Cierre de Minas, descrita anteriormente, fue observado por el Poder Ejecutivo ${ }^{26}$ en tres artículos: artículo $12^{\circ}$ segundo párrafo, artículo $14^{\circ} \mathrm{y}$ artículo $15^{\circ 27}$. Es decir, en la Ley 28090 aprobada y promulgada, se eliminó el segundo párrafo del artículo $12^{\circ}$, y se eliminaron los artículos $14^{\circ}$ y $15^{\circ}$ en su totalidad. A continuación, se presenta el contenido de los artículos que fueron observados por el Poder Ejecutivo, así como las razones que sustentaron dicha decisión.

El artículo $14^{\circ}$ del Proyecto de Ley del Cierre de Minas pretendía incorporar un literal e) al tercer párrafo del artículo $18^{\circ}$ del Decreto Supremo 054-99-EF, Texto Único Ordenado de la Ley Impuesto a la Renta, por el cual se solicitaba la inafectación del Impuesto a la Renta de los intereses y/o las ganancias de capitales generados por las garantías ambientales ${ }^{28}$.

Según el Sr. Alejandro Oré Mora, presidente de la Comisión de Energía y Minas del Congreso de la República, en el Allanamiento sobre Cierre de Mina del año 2003, de no haberse observado el artículo $14^{\circ}$ del Proyecto de Ley en cuestión, se hubiera producido una pérdida de recaudación por este concepto y violentado el principio de igualdad consagrado en el inciso 2 ) del artículo $2^{\circ}$ de la Constitución Política del Perú de $1993^{29}$. Con respecto a esto último, según el Poder Ejecutivo, se hubiera establecido un trato discriminatorio frente a otros sectores de la economía nacional e, incluso, al interior del sector minero, en la medida en que existen contribuyentes que ya han iniciado operaciones y cuentan con contratos de estabilidad tributaria respecto al Impuesto a la Renta y que adicionalmente estarían obligados a tener su Plan de Cierre de Minas. En consecuencia, los beneficios tributarios adicionales que se pretendería otorgar no los alcanzaría, correspondiendo su goce exclusivamente a

23. Sociedad Nacional de Minería, Petróleo y Energía 2005.

24. La descripción de las etapas fue tomado de: CIDA - Proyecto de asistencia al sector público minero metalúrgico del Perú, Oficina de Evaluación Ambiental de British Columbia Golder Associates LTD 2002.

25. Grupo Consulcont S.A.C. y Smallvill S.A.C. "CIERRE DE MINA (Ley N² 28090 y su modificatoria Ley N² 28507)". En: http://www. smallvillsac.com/cierre.htm\#imp

26. Mediante Oficio $N^{\circ} 165-2003-P R$, de fecha 4 de agosto de 2003.

27. Congreso de la República $2003 b$.

28. Ibídem.

29. Ibídem. 
proyectos que se inicien en fecha posterior a la vigencia de la Ley $^{30}$.

De esta manera, el inciso 2) del artículo $2^{\circ}$ de la Constitución del Perú indica que toda persona tiene derecho «A la igualdad ante la ley. Nadie debe ser discriminado por motivo de origen, raza, sexo, idioma, religión, opinión, condición económica 0 de cualquiera otra índole ${ }^{31}$. Se entiende que la justificación expuesta por el $\mathrm{Sr}$. Mora para eliminar el artículo $14^{\circ}$ del Proyecto de Ley, se refiere a que, según el principio de igualdad tributaria, los contribuyentes con homogénea capacidad contributiva deben recibir el mismo trato legal y administrativo frente al mismo supuesto de hecho tributario ${ }^{32}$. Asimismo, se refiere a que el legislador, al otorgar un beneficio tributario, debe respetar el principio de igualdad en materia tributaria, ya que en el marco de una política fiscal racional, los beneficios deben justificarse en razones objetivas que promuevan el desarrollo de las actividades económicas ${ }^{33}$.

Por otro lado, el artículo $15^{\circ}$ del Proyecto de Ley de Cierre de Minas disponía la inclusión de un nuevo literal en el artículo $37^{\circ}$ de la Ley del Impuesto a la Renta, el cual señalaba lo siguiente: «Son deducibles las provisiones que se constituyan para la ejecución del Plan de Cierre de las operaciones de las empresas dedicadas a las actividades de extracción de recursos naturales de acuerdo con las normas específicas que promulgue la Autoridad Sectorial competente.

30 Asimismo, son deducibles en el ejercicio en que se incurran los gastos relacionados con la protección ambiental y la ejecución del Plan de Cierre, así como los gastos incurridos en la constitución de las garantías, las primas de seguro y demás, requeridos para la formalización de las garantías exigidas. Los importes a los que se refiere el presente inciso, que al finalizar la ejecución del Plan de Cierre no hubieren sido utilizados, se considerarán beneficios gravables con el Impuesto a la Renta» ${ }^{34}$.

Este artículo fue observado por el Poder Ejecutivo, y dicha observación se centra en dos aspectos principales. "La deducción de los gastos, distintos a la provisión, no se encuentra expresamente prohibida por la Ley del Impuesto a la Renta, por lo que resulta posible deducir tales gastos siempre que constituyan gastos propios de la actividad empresarial.

En cuanto a la provisión, se señala que no es apropiada su inclusión, entre otras razones por cuanto se quebrantaría el compromiso del país de no introducir medidas que impliquen menor recaudación, y por cuanto se generaría doble deducción compuesta por los propios gastos efectuados y por las provisiones del mismo ejercicio en que se efectuaron los gastos» ${ }^{35}$.

Durante el Allanamiento sobre Cierre de Minas, en el Congreso de la República, el Sr. Oré Mora señaló que la Comisión de Energía y Minas coincidía con el Poder Ejecutivo en que, en efecto, el permitir la deducción de la Provisión por Cierre de Minas generaría doble deducción, la cual estaría compuesta por los propios gastos ejecutados, los cuales ya están amparados por la Ley del Impuesto a la Renta, y las provisiones del ejercicio que se constituyen para el establecimiento de las garantías ambientales, las que estarían amparadas por la Ley del Cierre de Minas. Para la mencionada Comisión, esta situación generaría una doble deducción, por lo que consideró pertinente eliminar el artículo $15^{\circ 36}$.

Finalmente, el Poder Ejecutivo consideró pertinente excluir el segundo párrafo del artículo $12^{\circ}$, el cual también señalaba que era permitida la deducción de las provisiones del ejercicio del Cierre de Minas. De manera que, el artículo $12^{\circ}$ de la Ley de Cierre de Minas expresa únicamente

30. Ministerio de Economía y Finanzas 2003.

31. Congreso de la República del Perú 1993.

32. Robles Moreno 2008.

33. Estudio Bravo Sheen Abogados 2008.

34. De Orbegoso 2004.

35. Ibídem.

36. Congreso de la República $2003 b$ 
lo siguiente: «La provisión contable del ejercicio destinada a cubrir el costo del Plan de Cierre de Minas deberá registrarse a partir del ejercicio contable en que el titular minero deba constituir garantía, según el cronograma aprobado por la autoridad competente ${ }^{37}$. Por lo tanto, la indicada norma regula el momento en que debe contabilizarse la provisión para efectos contables; sin embargo, no regula que la provisión tenga también efecto tributario ${ }^{38}$.

\section{Tratamiento contable de la provisión por cierre de minas}

\section{Tratamiento contable - financiero}

Financieramente, el tratamiento contable del Cierre de minas se encuentra regulado por las Normas Internacionales de Contabilidad (IAS, por sus siglas en inglés) así como por los Normas de Contabilidad Financiera (FAS, por sus siglas en inglés) que representan los principios contables norteamericanos (USGAAP's). Estas normas atienden a diferentes puntos como por ejemplo la determinación del monto a ser registrado, el registro inicial con las cuentas contables que deben ser impactadas, las actualizaciones al registro, entre otros; que serán detallados a continuación ${ }^{39}$.

Normas involucradas en el tratamiento contable - financiero

Se destacan las siguientes normas:

- NIC 16 - Inmuebles, Maquinaria y Equipos (revisada en diciembre de 2003)

- NIC 37 - Provisiones, Pasivos Contingentes y Activos Contingentes

- FAS 143 - Accounting for Asset Retirement Obligations

\section{Reconocimiento inicial}

El Cierre de Minas debe ser reconocido en el Balance General como un activo de larga duración (activo) cuya contrapartida es una provisión (pasivo).

Para el registro del activo de larga duración, se toma como sustento la NIC 16 - Inmuebles, Maquinaria y Equipos que establece en el párrafo 16, sobre los componentes del costo, que:

«El costo de los elementos de propiedades, planta y equipo comprende:

(c) [...] la estimación inicial de los costos de desmantelamiento o retiro del elemento, así como la rehabilitación del lugar sobre el que se asienta, cuando constituyan obligaciones en las que incurre la entidad como consecuencia de utilizar el elemento durante un determinado periodo, con propósitos distintos del de la producción de inventarios durante tal periodo ${ }^{40}$.

Cabe resaltar dos conceptos incluidos en la norma que hacen referencia a lo que se considera dentro del concepto de Cierre de Minas. Por un lado, que (i) dichos costos hayan constituido obligaciones; así como (ii) la inclusión de los costos de retiro y rehabilitación dentro de los activos constituidos por la entidad.

(i) La obligación nace de la Ley 28090 que indica que:

«Los titulares de la actividad minera, están obligados a:

\} Implementar un Plan de Cierre de Minas planificado desde el inicio de sus actividades ${ }^{41}$.

(ii) Los costos de retiro y rehabilitación quedan especificados en el Reglamento de la Ley 28090 puesto que para la elaboración del Plan de Cierre de Minas se tendrá en cuenta lo siguiente:

«Debe incluir las medidas y presupuesto necesarios para rehabilitar el lugar en el que se han desarrollado actividades mineras, asegurar la estabilidad física y química de los residuos y componentes mineros

37. Congreso de la República del Perú 2003a.

38. De Orbegoso, 2004.

39. Ver ANEXO 1: Ejemplo del Reconocimiento y Medición de la Provisión por Cierre de Minas.

40. IASB 2004a.

41. Congreso de la República del Perú 2003a. 
susceptibles de generar impactos negativos y establecer condiciones adecuadas para que el desarrollo y término del proyecto minero, sea acorde con los mandatos establecidos en la legislación vigente».

En adición, considerando la contrapartida del reconocimiento contable inicial, el pasivo queda constituido por una provisión, que en este caso está normada por la NIC 37 - Provisiones, pasivos contingentes y activos contingentes. En el párrafo 14, esta NIC determina que:

«Debe reconocerse una provisión cuando se den las siguientes condiciones:

(a) la entidad tiene una obligación presente (ya sea legal o implícita) como resultado de un suceso pasado;

(b) es probable que la entidad tenga que desprenderse de recursos, que incorporen beneficios económicos para cancelar tal obligación; y

(c) puede hacerse una estimación fiable del importe de la obligación $»^{42}$.

Así como en lo planteado por la NIC 16, la obligación queda determinada por la Ley 28090; por otra parte, el desprendimiento de recursos para cancelar la obligación y la estimación fiable del importe quedan detallados por las garantías y presupuestos que deben ser establecidos por el titular de la actividad.

De esta manera, el reconocimiento inicial del Cierre de Minas es el siguiente:

\section{$X$ \\ (Debe - Activo) Costo de Cierre de Minas \\ (Haber - Pasivo) Provisión por Cierre de Minas}

\section{Medición del reconocimiento inicial}

Una vez determinado el reconocimiento contable del Cierre de Minas, es necesario medir el monto por el cual se registrará dicha obligación. Es así como la FAS 143 - Accounting for Asset Retirement Obligations, en el párrafo 3, determina que:
«Una entidad reconocerá el valor justo de un pasivo de una obligación de retiro de activos en el periodo en el cual es incurrido si una estimación razonable del valor justo puede ser hecha. Si una estimación razonable de valor justo no puede ser hecha en el período en que la obligación de retiro de activo es incurrida, el pasivo será reconocido cuando una estimación razonable de valor justo puede ser hecha ${ }^{43}$.

Asimismo, esta norma indica que para determinar el valor razonable de la obligación pueden utilizarse diversas técnicas en ausencia de un mercado activo que las valorice, por lo que propone la técnica de valuación del Valor Presente. El párrafo 7 de la norma determina que:

«El valor justo de un pasivo por una obligación de retiro de activos es la cantidad por la cual aquel pasivo podría ser instalado en una transacción corriente entre partes dispuestas, es decir, otras distintas que una forzada o de liquidación. Los precios de mercado cotizados en mercados activos son mejores pruebas de valor justo y serán usados como la base para la medida, de ser disponible. Si los precios de mercado cotizados no se encontraran disponibles, la estimación del valor justo se debe basar en la mejor información disponible en dicha circunstancia, incluyendo los precios de pasivos similares y el resultado de la técnica de valor presente u otra técnica de valuación» ${ }^{44}$.

Por otra parte, el Reglamento de la Ley 28090 establece en su artículo $42^{\circ}$ los componentes del presupuesto, entre los cuales se detalla:

«El presupuesto del Plan de Cierre de Minas se calcula sobre la base del monto total estimado conforme a lo señalado en el artículo anterior e incluye los siguientes aspectos:

42.1 El valor presente neto actualizado del Plan de Cierre de Minas, considerando

\footnotetext{
42. IASB 2004b.

43. FASB 2001.

44. Ibídem.
} 
como base la fecha de ejecución de las medidas consideradas en dicho Plan $[\ldots]{ }^{45}$.

De esta manera, la medición del reconocimiento inicial se hará considerando la técnica del valor presente, como lo establece el Reglamento de la Ley 28090 y la FAS 143; asimismo, se tomarán en cuenta las siguientes condiciones descritas en la FAS 143:

«Apéndice A: Directrices de
Implementación

A20. [...] Los flujos de caja usados en estimados de valor razonable deben incorporar supuestos que los participantes del mercado podrían usar en sus estimados para reflejar los costos y la oportunidad para el retiro de activos similares, con el objetivo de determinar el monto que un tercero requeriría para el retiro de dichos activos, se deberían incorporar supuestos como:

(a)El costo que un tercero incurriría en el retiro del activo.

(b)Otros montos que un tercero podría incluir en determinar el monto final para liquidar el activo, incluyendo inflación, costo de equipos, avances en tecnología.

(c) La extensión en la cual variaría el costo u oportunidad del tercero bajo diferentes escenarios futuros y la probabilidad de dichos escenarios.

(d)El precio que un tercero demandaría recibir por enfrentar incertidumbre y circunstancias inesperadas inherentes en la obligación, referidas como riesgo de mercado $[\ldots]{ }^{46}$.

Asimismo, para el descuento de los flujos, el párrafo 9 de la misma norma señala que:

«[...] Cuando una entidad usa el enfoque de flujo de caja esperado, debe descontar los flujos de caja utilizando una tasa libre de riesgo y ajustada por los efectos de su situación de crédito» ${ }^{47}$.

\section{Reconocimiento y medición en periodos posteriores}

Al ser el Cierre de Minas registrado como un activo de larga duración, debe ser depreciado periódicamente de acuerdo con lo establecido por la NIC 16, en el párrafo 6 :

«[...] Importe depreciable es el costo de un activo, o el importe que lo haya sustituido, menos su valor residual.

Depreciación es la distribución sistemática del importe depreciable de un activo a lo largo de su vida útil $[\ldots] »^{48}$

Asimismo, el método de depreciación que la entidad utilizará para depreciar el activo constituido por el Cierre de Minas puede ser por línea recta (proporción igual periodo a periodo) o de acuerdo con las unidades producidas, que en el caso de la minería es un factor determinado por la división de las unidades producidas entre la suma de los recursos y reservas del periodo; así como lo establece la NIC 16 en el párrafo 62:

"Pueden utilizarse diversos métodos de depreciación para distribuir el importe depreciabledeunactivodeformasistemática a lo largo de su vida útil. Entre los mismos se incluyen el método lineal, el método de depreciación decreciente y el método de las unidades de producción. La depreciación lineal dará lugar a un cargo constante a lo largo de la vida útil del activo, siempre que su valor residual no cambie [...] El método de las unidades de producción dará lugar a un cargo basado en la utilización o producción esperada. La entidad elegirá el método que más fielmente refleje el patrón esperado de consumo de los beneficios económicos futuros incorporados al activo. Dicho método se aplicará uniformemente

45. Congreso de la República del Perú 2005.

46. FASB 2001.

47. Ibídem.

48. IASB 2004a. 
en todos los periodos, a menos que se haya producido un cambio en el patrón esperado de consumo de dichos beneficios económicos futuros." 49

Por lo tanto, la depreciación del activo de larga duración por Cierre de Minas quedará reflejada en la contabilidad, periodo a periodo de acuerdo con el siguiente asiento:

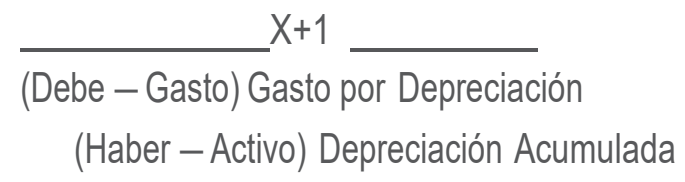

Por otra parte, conforme van pasando los años de vida de la unidad minera sujeta al Cierre de Minas, el valor presente de los flujos futuros de caja que sirven como base para el registro inicial de la provisión realizada se va actualizando.

El párrafo 13 y 14 de la FAS 143 - Accounting for Asset Retirement Obligations, establece que:

"13. En periodos subsecuentes a la medición inicial, la entidad debe reconocer, periodo a periodo, los cambios en el pasivo por obligación de retiro de activos que resultan de (a) el paso del tiempo y (b) las revisiones del tiempo o monto de la estimación original de los flujos de caja descontados. La entidad debe medir e incorporar los cambios debidos al paso del tiempo en el monto del pasivo antes de medir los cambios resultantes de la revisión del tiempo o monto de la estimación de los flujos de caja descontados.

14. La entidad deberá medir los cambios en el pasivo por obligación de retiro de activos debidos al paso del tiempo aplicando un método de asignación de interés al monto del pasivo al inicio del periodo. La tasa de interés usada para medir ese cambio debe ser la tasa libre de riesgo ajustada por riesgo que existía cuando el pasivo, o porción de eso, fue inicialmente medido. Ese monto debe ser reconocido como un gasto clasificado como un artículo operativo en el Estado de Ganancias y Pérdidas, desde ahora referido como un aumento del gasto [...]" 50 .

Por lo tanto, esta actualización de la medición inicial de la Provisión por Cierre de Minas quedaría reflejada en la contabilidad, periodo a periodo, con el siguiente asiento:

$\overline{\text { (Debe }- \text { Gasto) Gasto Financiero }}^{\text {X+2 }}$
$\quad$ (Haber - Pasivo) Provisión por Cierre de Minas

\section{Registro final de la Provisión por Cierre de Minas}

Al final de la vida útil de la unidad minera, la entidad no poseerá más el activo de larga duración por Cierre de Minas, pues este habrá ido consumiéndose, periodo a periodo, de acuerdo con lo planificado para la depreciación. Sin embargo, aún se cuenta con la contrapartida por el lado del pasivo, constituida por la Provisión por Cierre de Minas, la cual ha venido actualizándose periodo a periodo de acuerdo con la medición del valor presente de los flujos futuros de caja. De esta manera, la forma de eliminar esta Provisión por Cierre de Minas será registrándola contablemente con un cargo por la totalidad de la provisión y un abono por concepto de los trabajos realizados por pagar, asignaciones de gasto generales y de equipos, entre otros.

Por lo tanto, el registro final por concepto de Cierre de Minas, quedaría reflejado en la contabilidad con el siguiente asiento:

$$
\begin{aligned}
& \text { X+3 } \\
& \begin{array}{l}
\text { (Debe - Pasivo) Provisión por Cierre de Minas } \\
\text { (Haber - Pasivo) Cuentas por pagar diversas }
\end{array}
\end{aligned}
$$

\section{Tratamiento contable - tributario}

Con respecto a la deducibilidad para efectos del Impuesto a la Renta, el tratamiento de la Provisión por Cierre de Minas no es considerado por la Ley 
28090 - Ley que regula el Cierre de Minas y su Reglamento, ni regulado específicamente por la Ley del Impuesto a la Renta.

El artículo $37^{\circ}$ de la Ley del Impuesto a la Renta señala que "A fin de establecer la renta neta de tercera categoría se deducirá de la renta bruta los gastos necesarios para producirla y mantener su fuente, así como los vinculados con la generación de ganancias de capital, en tanto la deducción no esté expresamente prohibida por esta ley, en consecuencia son deducibles [...]"51

Mientras que el artículo $44^{\circ}$ señala que "No son deducibles para la determinación de la renta imponible de tercera categoría: [...] f) Las asignaciones destinadas a la constitución de reservas o provisiones cuya deducción no admite esta ley". ${ }^{52}$

Como se menciona en el primer párrafo, la Ley del Impuesto a la Renta no regula específicamente el tratamiento tributario de la provisión. Sin embargo, en la práctica, la Provisión por Cierre de Minas no es permitida como gasto deducible para efectos del Impuesto a la Renta porque se considera como provisión genérica. Es decir, que el gasto por depreciación del ejercicio generado por el activo de larga duración, correspondiente a la Provisión por Cierre de Minas, de acuerdo con lo explicado anteriormente, se debe reparar para efectos del cálculo del Impuesto a la Renta.

Esto último se debe a que el activo que se genera por el retiro por Cierre de Minas obedece estrictamente a una evaluación de carácter financiero. Por lo tanto, no es un activo fijo ante la apreciación de la Administración Tributaria y, por ende, la depreciación que se reconoce por dicho activo tampoco es aceptada como tal por dicha entidad.

\section{Fiscalización tributaria de la Provisión por Cierre de Minas ${ }^{53}$}

En este acápite, se busca explicar en qué consistiría la fiscalización tributaria de una unidad minera por concepto de la Provisión por Cierre de Minas por parte de la Administración Tributaria, en el supuesto caso de que la depreciación que se origina producto del reconocimiento del retiro por Cierre de Minas como un activo de larga duración sea aceptado como gasto deducible para el cálculo de la renta neta imponible.

En primer lugar, la Administración Tributaria tendría que reconocer el activo de larga duración determinado desde el punto de vista financiero $y$, por ende, tendría que establecer en el Reglamento de la Ley del Impuesto a la Renta un método de depreciación aplicable a dicho activo con el fin de que la unidad minera pueda tener un referente que se pueda aplicar a los fines de la determinación del impuesto y para los demás efectos previstos en normas tributarias.

Por otro lado, una vez establecido lo mencionado en el párrafo anterior, cuando efectivamente se lleve a cabo una fiscalización por el concepto de Provisión por Cierre de Minas, los auditores de la Administración Tributaría evaluarían, a grandes rasgos, los siguientes aspectos:

- Como existen gastos reales efectuados durante el Cierre Progresivo que sí son considerados deducibles, los auditores pedirían el sustento de dichos gastos, por lo que los comprobantes de pago relacionados deberían estar disponibles para su revisión. Esto último ya quedaría bajo la entera responsabilidad de la unidad minera que esté siendo objeto de la fiscalización.

- Asimismo, como el activo de larga duración ya sería reconocido por la Administración Tributaria, entonces todo lo relacionado con el cálculo y el reconocimiento de la depreciación tendría que ser registrado por la unidad minera en el Registro de Activo $\mathrm{Fijo}^{54}$. De esta manera, los auditores solicitarían este registro y sobre la información contenida en él, relacionada al activo de larga duración, evaluarían

51. Congreso de la República del Perú. "TuO de la Ley del Impuesto a la Renta". Perú. Capítulo VI, Artículo 37.- (56).

52. Congreso de la República del Perú. "TUO de la Ley del Impuesto a la Renta". Perú. Capítulo VI, Artículo 44.

53. Información obtenida de un ex empleado de la Superintendencia Nacional de Administración Tributaria.

54. Especificado por la Resolución de Superintendencia No 234-2006/SUNAT, formato 7.1. 
la existencia de diferencias temporales que se pudiesen producir en caso se haya determinado para la mina una vida útil diferente a la hallada como resultado de la aplicación del método de depreciación estipulado por la Administración Tributaria.

\section{Prácticas contables de la industria minera}

El tratamiento contable - financiero y contable - tributario de la Provisión por Cierre de Minas explicado en los subcapítulos anteriores refleja, desde el punto de vista de la normatividad financiera y tributaria respectivamente, lo que en la práctica deberían efectuar las empresas que pertenecen a la industria minera. Es así que dentro de esta industria, las empresas tienen como práctica contable común registrar como Provisión por Cierre de Minas un importe que refleje el total de las erogaciones a efectuarse en cada una de las etapas del Cierre de Minas (Cierre Progresivo, Cierre Final y Post Cierre), necesarias para cumplir con el Plan de Cierre de Minas aprobado por la autoridad competente. De esta manera, el monto provisionado no necesariamente coincide con el importe consignado como garantía ante el Estado para el cumplimiento de dicho plan ${ }^{55}$. Posteriormente, el importe de esta provisión es ajustado (reducido) a medida que se efectúen los gastos propios del Cierre de Minas durante el periodo de Cierre Progresivo, debido a que dichos gastos sí son deducibles para efectos del cálculo del Impuesto a la Renta. Asimismo, este pasivo también se debe actualizar, hacia arriba o hacia abajo, debido a los cambios que experimenta el dinero en el tiempo.

Por otro lado, cuando se llegue al final de la vida útil de la unidad minera, que generalmente coincide con el periodo en el que la empresa ya no genera ingresos debido al término de la operación de la mina (inicio de las etapas de Cierre
Final y Post Cierre), el monto que todavía esté registrado en el Balance General como Provisión por Cierre de Minas ${ }^{56}$ (pasivo) se extorna contra la cuenta Cuentas por pagar, constituyéndose como futuros desembolsos de efectivo que no serán utilizados tributariamente como gasto deducible para el cálculo del Impuesto a la Renta generado por dicha unidad minera porque esta no tendrá ingresos contra los cuales imputar la totalidad del gasto mencionado.

Sin embargo, como es común que las empresas pertenecientes a la gran minería posean más de una unidad minera, estas compañías suelen utilizar los futuros desembolsos de efectivo mencionados en el párrafo anterior como gasto deducible en otras unidades mineras de su propiedad que todavía se encuentren en la etapa de Cierre Progresivo ${ }^{57}$, a fin de reducir su base imponible como una estrategia de planeamiento tributario. Lo que se da en la práctica es que estas grandes empresas tratan a sus unidades mineras como centros de costos independientes a nivel de papeles de trabajo, pero al momento de armar los Estados Financieros las unidades mineras en cuestión ya no se tratan como centros de costos, sino que los resultados se consolidan. Asimismo, siempre va a quedar un importe no utilizado como gasto deducible por la empresa, correspondiente a la Provisión por Cierre de Minas de la unidad minera con la vida útil más larga.

Asimismo, está lógica de utilizar la pérdida de una unidad minera en otra cuya vida útil todavía exista, se da también en el caso de grupos económicos dedicados a la actividad minera, los cuales emplean la pérdida registrada por una de sus empresas en otra que esté registrando ganancias a través de la figura de fusión como parte de la reorganización de sociedades.

55. Generalmente, la provisión registrada es por un monto superior a la garantía, ya que según el artículo $11^{\circ}$ de la Ley 28090 - Ley que regula el Cierre de Minas, la garantía debe cubrir solamente los costos de las medidas de rehabilitación para los periodos de Cierre Final y Post Cierre.

56. Este importe solo debería reflejar los gastos a efectuarse durante las etapas de Cierre Final y Post Cierre.

57. Es decir, que aún tengan vida útil o generen ingresos. 


\section{Conclusiones}

Razones para considerar a la Provisión por Cierre de Minas como gasto deducible para efectos del Impuesto a la Renta:

Según lo tratado a lo largo del desarrollo del trabajo de investigación, se puede concluir que para considerar los gastos generados por la depreciación del activo constituido por Cierre de Minas como gastos deducibles para efectos del Impuesto a la Renta, se han considerado los siguientes argumentos que validan dicha propuesta:

(a)Vacíos en la legislación en materia tributaria: La Ley del Impuesto a la Renta no contiene una definición del concepto del activo de larga duración. Por ello, ante vacíos en la legislación en materia tributaria, los usuarios de la Ley deben tomar como base las Normas Internacionales de Contabilidad aprobadas en el Perú. ${ }^{58}$ De esta manera, es la NIC 16 la norma que define los costos a ser incluidos en un activo de larga duración. Así, esta NIC establece:

«El costo de los elementos de propiedades, planta y equipo comprende:

[...]

(c) la estimación inicial de los costos de desmantelamiento o retiro del elemento, así como la rehabilitación del lugar sobre el que se asienta, cuando constituyan obligaciones en las que incurre la entidad como consecuencia de utilizar el elemento durante un determinado periodo, con propósitos distintos del de la producción de inventarios durante tal periodo» ${ }^{59}$.

Asimismo, la NIC 16 establece que dichos activos deben ser depreciados de acuerdo con su vida útil; tema que sí es contemplado en la Ley del Impuesto a la Renta como un gasto deducible para el cálculo de dicho impuesto.

(b)Obligación de constituir garantías: La Ley 28090 - Ley que regula el Cierre de Minas, específicamente el artículo $11^{\circ}$.- Garantía Ambiental, obliga al titular minero a constituir garantías a favor de la autoridad competente para cubrir los costos de las medidas de rehabilitación para los periodos de Cierre Progresivo ${ }^{60}$, Cierre Final y Post Cierre, estando garantizado que el titular de la actividad minera vaya a incurrir en el gasto por Cierre de Minas (ya sea por la real ejecución del Plan de Cierre o por la ejecución de la garantía destinada a cubrir dicho plan). Bajo estas circunstancia el titular minero no tiene incentivo para sobrevaluar las provisiones destinadas a cubrir los gastos en mención, ya que ello traería consigo la obligación de incrementar la garantía ambiental en igual proporción, lo cual generaría un gasto adicional que podría llegar a ser mayor que cualquier ahorro tributario derivado de la deducción de la provisión misma (en caso estuviese permitida). Asimismo, el que la deducción de la Provisión por Cierre de Minas se encuentre limitada al monto cubierto por la garantía ambiental, asegura que dicha provisión sea estimada con sustento técnico suficiente y con una adecuada aproximación, debido a que es una Entidad Consultora inscrita en el Registro de Entidades Autorizadas a elaborar Planes de Cierre de Minas en el Sector Energía y Minas la que planifica detalladamente dicho cierre. Además, está el hecho de que el cálculo de la garantía en mención debe ser aprobado y controlado por la Dirección General de Asuntos Ambientales (DGAA). Por otro lado, cabe mencionar que dicha garantía debe ajustarse anualmente conforme vayan variando las condiciones

58. Información obtenida a través de entrevista realizada a Beatriz de la Vega, Gerente de Impuestos de Ernst \& Young. 13.10.2008.

59. ASB 2004a.

60. El artículo $48^{\circ}$ del Reglamento para el Cierre de Minas establece que el titular de la actividad minera no está obligado a constituir garantías que cubran los gastos por las actividades de la etapa de Cierre Progresivo, ya que dicha decisión queda a su elección. 
de cada operación minera, con lo cual el importe de la provisión también deberá ser revisado necesariamente con esa periodicidad.

(c) Cumplimiento del principio de Causalidad: El artículo $37^{\circ}$ de la Ley del Impuesto a la Renta establece el principio de Causalidad, según el cual, para efectos de la determinación del impuesto, resultan deducibles todos los gastos necesarios para generar renta gravada o para mantener su fuente. Dentro de estos gastos, ineludiblemente se debe considerar a la Provisión por Cierre de Minas, la cual afecta resultados bajo la forma de la depreciación del activo de larga duración constituido por Cierre de Minas, ya que dicha provisión se reconoce como consecuencia de cumplir con la obligación indispensable, normada por ley, de establecer las garantías ante el Estado para el cumplimiento del Plan de Cierre de Minas. Es así que recién después de llevar a cabo este requisito, los titulares de la actividad minera pueden iniciar operaciones. De esta manera, lo indicado sería que la provisión contable destinada a cubrir el costo del Plan de Cierre también sea aceptada como gasto deducible a efectos tributarios.

(d)Descarte del argumento de doble deducción: No se produciría una doble deducción, compuesta por los propios gastos efectuados y por las provisiones del mismo ejercicio en que se efectuaron los gastos, ya que dichas provisiones se devengarían bajo la forma de depreciaciones, no reconociéndose otros gastos sino aquellos en los que efectivamente se incurrió durante la etapa de Cierre Progresivo, los cuales sí son reconocidos y aceptados como importes deducibles para calcular la renta neta imponible.
(e)Posibilidad de la realización de una fiscalización de la Provisión por Cierre de Minas: De acuerdo con lo señalado en el acápite 5.2.1 Fiscalización de la Provisión por Cierre de Minas, de reconocerse el retiro por Cierre de Minas como activo de larga duración, así como la depreciación de este como gasto deducible, la fiscalización por este concepto no requeriría de la realización de actividades o la utilización de recursos adicionales a los que normalmente se consideran en cualquier fiscalización. En todo caso, lo más complicado respecto al tema sería la determinación por parte de la Administración Tributaria de un método de depreciación a ser aplicable al activo de larga duración que refleje de manera adecuada la vida útil de la unidad minera, es decir, que contemple la posibilidad de descubrir más recursos a los planificados en un inicio durante la etapa pre-operativa (de exploración).

(f) Promoción de la inversión en el sector: Si bien el Estado obtendría una menor recaudación, el fin de permitir la deducción de la Provisión por Cierre de Minas (bajo la forma de depreciación del activo de larga duración) sería establecer medidas que promuevan la posibilidad de una inversión segura, en tanto existan reglas claras de qué se podrá o no deducir, así como una inversión adecuada, en la medida que el Estado la reconozca y la relacione con el proceso generador de renta, no solo para la empresa misma sino también para el país ${ }^{61}$.

De esta manera, son varias las razones por las cuales la legislación en materia tributaria podría aceptar a la Provisión por Cierre de Minas como gasto deducible, con lo cual se descartan algunos de los motivos por los cuales se rechazó en un inicio su incorporación dentro de la Ley

61. Este es uno de los argumentos establecidos por la Sociedad Nacional de Minería, Petróleo y Energía (SNMPE) en la carta firmada por el presidente de dicha sociedad, José Miguel Morales Dasso, al presidente de la Comisión de Energía y Minas del Congreso de la República, congresista Alejandro Oré, ante las observaciones efectuadas por el Poder Ejecutivo y dicha comisión al Proyecto de Ley de Cierre de Minas. Lima, 28 de agosto de 2003. 
del Impuesto a la Renta. Asimismo, tal como se ejemplificó en el capítulo anterior, existen varios países que sí aceptan su deducción para efectos del cálculo de la renta neta imponible, apoyando la posición final de la presente investigación con respecto a este tema y sirviendo como base de comparación para futuras evaluaciones por parte de la Administración Tributaria con el fin de que esta provisión pueda ser considerada como gasto deducible.

\section{ANEXO 1: Ejemplo del reconocimiento y medición de la Provisión por Cierre de Minas $^{62}$}

Una entidad peruana completa la construcción y pone en servicio una unidad minera el 1 de enero de 2003. Esta entidad tiene la obligación legal de desmantelar y remover la unidad minera al final de su vida útil, la cual ha sido estimada en 10 años. Basados en los requerimientos de la FAS 143, el 1 de enero de 2003, la entidad reconoce un pasivo por la obligación del retiro de los activos y capitaliza el monto por el costo de la obligación del retiro de los activos. La entidad estima el valor razonable inicial del pasivo usando la técnica del valor presente esperado. Las suposiciones significativas utilizadas en la estimación del valor razonable son las siguientes:

(a)Los costes de trabajo están basados en salarios de mercado corrientes requeridos para alquilar a contratistas para desmontar y remover la unidad minera. La entidad asigna evaluaciones de probabilidad a una rango de estimaciones de flujo de caja, de la siguiente forma:

\begin{tabular}{|c|c|c|}
\hline $\begin{array}{c}\text { Flujo de Caja } \\
\text { Estimado }\end{array}$ & $\begin{array}{c}\text { Determinación } \\
\text { de la } \\
\text { Probabilidad }\end{array}$ & $\begin{array}{c}\text { Flujos de Caja } \\
\text { Esperados }\end{array}$ \\
\hline S/. 100,000 & $25 \%$ & S/. 25,000 \\
S/. 125,000 & $50 \%$ & S/. 62,500 \\
S/. 175,000 & $25 \%$ & S/. 43,750 \\
\cline { 3 - 3 } & & S/. 131,250 \\
\hline
\end{tabular}

(b)Los estimados de la entidad asignan gastos generales y de equipos utilizando una tasa que aplica a los costos de trabajo para precios de transferencia (80\%). La entidad no tiene razón para creer que estas tasas para los gastos difieren de aquellas usadas por contratistas en la industria.

(c) Un contratista, normalmente adiciona un margen de trabajo y asigna costos internos para determinar un margen de ganancia en el trabajo. La tasa usada (20\%), representa el entendimiento de la entidad de la utilidad obtenida por los contratistas de la industria por el desmantelamiento y remoción de la unidad minera.

(d)Un contratista normalmente exigiría y recibiría una prima (la prima de riesgos de mercado) para llevar la incertidumbre y circunstancias imprevisibles inherentes en «el cierre» al precio de hoy para un proyecto que no ocurrirá durante 10 años. La entidad estima que la cantidad de aquella prima será del $5 \%$ de los flujos de caja estimados ajustados por inflación.

(e)La tasa libre de riesgo el 1 de enero de 2003 es de $5 \%$. La entidad ajusta esa tasa con $3.5 \%$ para reflejar el efecto de su situación crediticia. Entonces, la tasa libre de riesgo ajustada por crédito será de $8.5 \%$.

(f) La entidad asume una tasa de inflación de $4 \%$ por el periodo de 10 años.

El 31 de diciembre de 2012, la entidad ejecuta su obligación para el retiro de activos mediante el uso de una fuerza de trabajo interna a un costo de $S / .351,000$. Asumiendo que no hay cambios en los flujos de caja utilizados para la estimación de la obligación durante los 10 años, la entidad reconocerá una ganancia de S/. 89,619 por la ejecución de la obligación:

62. Ejemplo adaptado del Ejemplo 1 del Apéndice C: Ejemplos Ilustrativos - Reconocimiento y medición de Provisiones en la FAS 143 - Accounting for Asset Retirement Obligations. 


\begin{tabular}{|l|r|}
\hline Trabajos & S/. 195,000 \\
\hline $\begin{array}{l}\text { Asignación de gastos } \\
\text { generales y de equipos (80\%) }\end{array}$ & S/. 156,000 \\
\hline $\begin{array}{l}\text { Total de gastos } \\
\text { incurridos }\end{array}$ & S/. 351,000 \\
\hline $\begin{array}{l}\text { Provisión por Cierre } \\
\text { de Minas (ARO }{ }^{63} \text { ) }\end{array}$ & S/. 440, 619 \\
\hline $\begin{array}{l}\text { Ganancia en ejecución } \\
\text { de la obligación }\end{array}$ & S/. 89, 619 \\
\hline
\end{tabular}

Medición inicial de la Provisión por Cierre de Minas al 1 de enero de 2003:

\begin{tabular}{|l|c|}
\hline \multirow{2}{*}{ Costos de trabajo esperados } & $\begin{array}{c}\text { Flujos de } \\
\text { caja }\end{array}$ \\
\hline Asignación de gastos generales y de equipos (80\% x S/. 131,250) & S/. 131,250 \\
\hline Adicional del Contratista (20\% x [S/. 131,250 + S/. 105,000]) & S/. 105,000 \\
\hline Flujos de Caja Esperado antes del ajuste por inflación & S/. 47,250 \\
\hline Factor de inflación asumiendo una tasa de 4\% para 10 años & S/. 283,500 \\
\hline Flujos de Caja Esperado ajustado por inflación & 1.4802 \\
\hline Prima de riesgo de mercado (5\% x S/.419,637) & S/. 419,637 \\
\hline Flujos de Caja Esperados ajustados por riesgo de mercado & S/. 20,980, 40,619 \\
\hline $\begin{array}{l}\text { Valor Presente utilizando la tasa libre de riesgo ajustada por } \\
\text { crédito de 8.5\% para 10 años }\end{array}$ & S/. 194,879 \\
\hline
\end{tabular}

Método de asignación de intereses:

\begin{tabular}{|c|c|c|c|}
\hline Año & $\begin{array}{c}\text { Saldo del } \\
\text { pasivo al } 01.01\end{array}$ & Aumento & $\begin{array}{c}\text { Saldo del } \\
\text { pasivo al } 31.12\end{array}$ \\
\hline 2003 & S/. 194,879 & S/. 16,565 & S/. 211,444 \\
\hline 2004 & SI. 211,444 & S/. 17,973 & SI. 229,417 \\
\hline 2005 & SI. 229,417 & S/. 19,500 & SI. 248,917 \\
\hline 2006 & SI. 248,917 & S/. 21,158 & SI. 270,075 \\
\hline 2007 & SI. 270,075 & S/. 22,956 & SI. 293,031 \\
\hline 2008 & SI. 293,031 & SI. 24,908 & SI. 317,939 \\
\hline 2009 & S/. 317,939 & S/. 27,025 & SI. 344,964 \\
\hline 2010 & SI. 344,964 & S/. 29,322 & SI. 374,286 \\
\hline 2011 & S/. 374,286 & S/. 31,814 & S/. 406,100 \\
\hline 2012 & S/. 406,100 & S/. 34,519 & SI. 440,619 \\
\hline
\end{tabular}

63. ARO: Asset Retirement Obligation. 
El cronograma de gastos:

\begin{tabular}{|c|c|c|c|}
\hline Año & $\begin{array}{c}\text { Aumento del } \\
\text { gasto }\end{array}$ & $\begin{array}{c}\text { Gasto por } \\
\text { depreciación }\end{array}$ & $\begin{array}{c}\text { Total del } \\
\text { gasto }\end{array}$ \\
\hline 2003 & S/. 16,565 & S/. 19,488 & S/. 36,053 \\
\hline 2004 & S/. 17,973 & S/. 19,488 & S/. 37,461 \\
\hline 2005 & S/. 19,500 & S/. 19,488 & S/. 38,988 \\
\hline 2006 & S/. 21,158 & S/. 19,488 & SI. 40,646 \\
\hline 2007 & S/. 22,956 & S/. 19,488 & SI. 42,444 \\
\hline 2008 & SI. 24,908 & S/. 19,488 & Sl. 44,396 \\
\hline 2009 & S/. 27,025 & S/. 19,488 & S/. 46,513 \\
\hline 2010 & S/. 29,322 & S/. 19,488 & SI. 48,810 \\
\hline 2011 & S/. 31,814 & S/. 19,488 & S/. 51,302 \\
\hline 2012 & S/. 34,519 & Sl. 19,488 & S/. 54,007 \\
\hline
\end{tabular}

Asientos de diario:

\subsubsection{3}

Activos de Larga Duración (costo de retiro de activos)

S/. 194,879

Provisión por Cierre de Minas

S/. 194,879

Por el registro inicial del valor razonable de la provisión por Cierre de Minas

\subsubsection{3- 2012}

Gasto por Depreciación (costo de retiro del activo)

S/. 19,488

Depreciación Acumulada

Por el registro de la depreciación en línea recta del costo de retiro de activos

Aumento del Gasto (Financiero)

Según cronograma

Provisión por Cierre de Minas

Según cronograma

Por el registro del aumento en el Gasto por Cierre de Minas

\subsubsection{2}

Pasivo por Cierre de Minas

S/. 440,619

Trabajos por pagar

S/. 195,000

Asignación de gastos generales y de equipos (80\%)

S/. 156,000

Ganancia por establecimiento de la Provisión por C.M.

S/. 89,619

Por el registro de la realización de la Provisión por Cierre de Minas 


\section{BIBLIOGRAFÍA}

BIBLIOTECA DE LA UNMSM Pedro Zulen

2008 Guía ambiental para el cierre y abandono de minas. En: http://biblioteca.unmsm. edu.pe/redlieds/Recursos/archivos/ MineriaDesarrolloSostenible/Cierreminas/ cierreabandono.pdf. Consultada en 2008

CIDA - PROYECTO DE ASISTENCIA AL SECTOR PÚBLICO MINERO METALÚRGICO DEL PERÚ, OFICINA DE EVALUACIÓN AMBIENTAL DE BRITISH COLUMBIA GOLDER ASSOCIATES LTD.

2002 Guía para la elaboración y revisión de planes de Cierre de Minas. Marzo de 2002. Lima, Perú.

\section{CONGRESO DE LA REPÚBLICA DEL PERÚ}

2008 TUO de la Ley del Impuesto a la Renta. Lima. Perú.

2006 «DS 035-2006-EM - Modifican el DS 033-2005EM, que aprueba el Reglamento de la Ley que regula el Cierre de Minas». 04 de julio de 2006. Lima, Perú.

2005 DS 033-2005-EM - Reglamento para el Cierre de Minas. 16 de agosto de 2005. Lima, Perú.

2003a Ley 28090 - Ley que regula el Cierre de Minas. Octubre de 2003. Lima, Perú.

2003b «Allanamiento sobre Cierre de Minas». Primera Legislatura Ordinaria del 2003, $17^{\mathrm{a}}$ sesión (vespertina). 9 de octubre de 2003. En: http:// www.congreso.gob.pe/congresista/2006/ jcarrasco/intervenciones/03-10-09-17S-PLOv. htm.

1993 Constitución Política de 1993. Título I: «De la persona y de la sociedad, Capítulo I: Derechos fundamentales de la persona, Artículo $2^{\circ}$, inciso 2)». En: http://www2.congreso. gob.pe/sicr/RelatAgenda/constitucion.nsf/ abre?CreateDocument.

DE ORBEGOSO, Fernando

2004 «Implicancias tributarias del Cierre de Minas». VI Simposium de Tributación y Regulación Mineroenergética. Noviembre de 2004. Perú.

ERNST \& YOUNG

2008 Entrenamiento de Industrias especializadas: Sesión 8: «Tratamiento contable de provisiones ambientales y Cierre de Minas». Junio de 2008. Perú.

ESTUDIO BRAVO SHEEN ABOGADOS

2008 «Avícolas: Igualdad y Tribunal Constitucional (TC)». En Portal Tributario: http://

www.portaltributario.com.pe/detalle. php?d=Njc1MA==.

FASB

2001

FAS 143 - Accounting for Asset Retirement Obligations. Junio de 2001.

GRUPO CONSULCONT S.A.C. Y SMALLVILL S.A.C.

2008 «CIERRE DE MINA (Ley N² 28090 y su modificatoria Ley $\left.N^{\circ} 28507\right) »$. En: http://www. smallvillsac.com/cierre.htm\#imp.

IASB

2004a NIC 16 - Inmuebles, maquinaria y equipos. Marzo de 2004

2004b IASB. NIC 37 - Provisiones, pasivos contingentes y activos contingentes. Marzo de 2004.

LA TORRE O., Gonzalo

2008 Regulación Tributaria del Cierre de Minas. Estudio Ferrero Abogados.

MALETTA, H

2004 Metodología de la producción científica. Instituto de Investigación en Ciencias Sociales IDICSO de la Universidad del Salvador. Buenos Aires.

MINISTERIO DE ECONOMÍA Y FINANZAS

2003 «Autógrafa de Ley que regula el Cierre de Minas». Oficio múltiple No 221-2003-SCM-PR.

\section{MINISTERIO DE ENERGÍA Y MINAS}

2006 (Nota de prensa) «Proponen alianzas estratégicas para su remediación. Hay 850 pasivos ambientales en el país, que son herencia de la vieja minería», junio de 2006. En: http:// www.minem.gob.pe/common/prensa/data/ notihoy1800.pdf.

\section{MINISTERIO DE ENERGÍA Y MINAS}

2008 Actividad Minera, Aspectos a tener en cuenta. En: http://www.minem.gob.pe/archivos/dgm/ inversiones/exposiciones/actividad_minera_ archivos/frame.htm.

MINISTERIO DE ENERGÍA Y MINAS, DIRECCIÓN GENERAL DE ASUNTOS AMBIENTALES MINEROS

2006 Guía para la elaboración de Planes de Cierre de Minas. Abril de 2006. Lima, Perú.

MORALES Dasso, José Miguel

2003 Carta firmada por el presidente de la Sociedad Nacional de Minería, Petróleo y Energía (SNMPE), José Miguel Morales Dasso, al presidente de la Comisión de Energía y Minas del Congreso de la República, congresista Alejandro Oré. Lima, 28 de agosto de 2003, Lima. 
REFLEXIÓN DEMOCRÁTICA

2005 «Análisis del Proyecto Aprobado de Ley General del Ambiente». Fecha de aprobación 23/06/2005. En: http://www.reflexiondemocratica.org.pe/ pdfs/leygeneralambiente.pdf.

ROBLES Moreno, Carmen del Pilar

2008 Reflexiones sobre Derecho Tributario y Derecho Constitucional. «Los Principios Constitucionales Tributarios»». 14 de setiembre de 2008. En: http://blog.pucp.edu.pe/item/30553igual.

SOCIEDAD NACIONAL DE MINERÍA, PETRÓLEO Y ENERGÍA

2005 «Plan de Cierre de Minas». En: Informe quincenal de la SNMPE, noviembre I, 2005. Perú.

SUNAT

2006 Resolución de Superintendencia № 234-2006/ SUNAT, formato 7.1 .

Fuentes primarias

w Raúl del Pozo. Senior Manager del área de Auditoría Financiera de la firma Ernst \& Young Perú. Entrevista realizada el 9 de octubre de 2008. w Víctor Camarena. Senior Manager del área de Auditoría Financiera de la firma Ernst \& Young Perú. Entrevista realizada el 9 de octubre de 2008.

w Patricia Ramírez. Gerente del área de Auditoría Financiera de la firma Ernst \& Young Perú. Entrevista realizada el 21 de octubre de 2008.

w Marcial García. Socio del área de Impuestos de la firma Ernst \& Young Perú. Entrevista realizada el 15 de octubre de 2008.

w Beatriz de la Vega. Gerente del área de Impuestos de la firma Ernst \& Young Perú. Entrevista realizada el 16 de octubre de 2008.

w José Luis Loayza. Analista de Tributos del área de Asuntos Fiscales del Grupo Repsol YPF. Entrevista realizada el 30 de octubre de 2008.

w Carlos Carpio. Jefe del área de Asuntos Fiscales del Grupo Repsol YPF. Entrevista realizada el 15 de noviembre de 2008. 